\title{
PERIODIC APPROXIMATION OF OSELEDETS SUBSPACES FOR SEMI-INVERTIBLE COCYCLES
}

\author{
LUCAS BACKES
}

\begin{abstract}
We prove that, for semi-invertible linear cocycles, Oseledets subspaces associated to ergodic measures may be approximated by Oseledets subspaces associated to periodic points.
\end{abstract}

\section{INTRODUCTION}

Since its introduction by Smale in [Sm67], the notion of hyperbolicity has played a major rule in the study of Dynamical Systems. One of the main features exhibited by such systems is the abundance of periodic points and, as a consequence, many of its interesting dynamical properties can be described in terms of the information given on such periodic points. For instance, it is known that cohomology classes of Hölder cocycles over hyperbolic systems are characterized by its information on periodic points (see for instance [Liv71, Liv72, Kal11, dLW10, Bac15, Sa15, BK16, KP16] and references therein), equilibrium states associated to different potentials coincide whenever those potentials have the same information on periodic points [Bow75] and the information carried by the Lyapunov exponents is concentrated on periodic points [Dai10, WS10, Kal11, Bac].

In this note we address the problem of extracting information from periodic points in the context of Oseledets splittings of semi-invertible linear cocycles. As a consequence of our main result we get that (see Section 2.4 for precise statements)

Theorem 1.1. If $f: M \rightarrow M$ is a homeomorphism satisfying the Anosov Closing property and $A: M \rightarrow M(d, \mathbb{R})$ is a $\alpha$-Hölder continuous map then the Oseledets subspaces of $(A, f)$ associated to ergodic measures can be approximated by Oseledets subspaces of $(A, f)$ associated to periodic points.

This problem was already addressed by [LLS09] in the context of $C^{1+r}$ nonuniformly hyperbolic systems with simple Lyapunov spectrum and was latter generalized by [LLS14] to any $C^{1+r}$ non-uniformly hyperbolic system (that is, with no simplicity assumption). While both works dealt only with the case of derivative cocycles with no zero Lyapunov exponents (which is a particular example of invertible cocycle) we treat the broader case of semi-invertible cocycles.

Date: April 26, 2018.

2010 Mathematics Subject Classification. Primary: 37H15, 37A20; Secondary: 37D25.

Key words and phrases. Semi-invertible linear cocycles, Oseledets subspaces, periodic points, approximation. 


\section{Statements}

Let $(M, d)$ be a compact metric space, $\mu$ a measure defined on the Borel sets of $(M, d)$ and $f: M \rightarrow M$ a measure preserving homeomorphism. Assume also that $\mu$ is ergodic.

2.1. Semi-invertible cocycles, Lyapunov exponents and Oseledets splittings. Given a measurable matrix-valued map $A: M \rightarrow M(d, \mathbb{R})$, the pair $(A, f)$ is called a semi-invertible linear cocycle (or just linear cocycle for short). Sometimes one calls linear cocycle (over $f$ generated by $A$ ), instead, the sequence $\left\{A^{n}\right\}_{n \in \mathbb{N}}$ defined by

$$
A^{n}(x)= \begin{cases}A\left(f^{n-1}(x)\right) \ldots A(f(x)) A(x) & \text { if } n>0 \\ I d & \text { if } n=0\end{cases}
$$

for all $x \in M$. The word 'semi-invertible' refers to the fact that the action of the underlying dynamical system $f$ is invertible while the action on the fibers given by $A$ may fail to be invertible. We refer to the Introduction of $[\mathrm{DrF}]$ for some interesting applications of semi-invertible cocycles.

Assuming $\int \log ^{+}\|A(x)\| d \mu(x)<\infty$, it was proved in [FLQ10] that there exists a full $\mu$-measure set $\mathcal{R}^{\mu} \subset M$, whose points are called $\mu$-regular points, such that for every $x \in \mathcal{R}^{\mu}$ there exist numbers $\lambda_{1}>\ldots>\lambda_{l} \geq-\infty$, called Lyapunov exponents, and a direct sum decomposition $\mathbb{R}^{d}=E_{x}^{1, A} \oplus \ldots \oplus E_{x}^{l, A}$ into vector subspaces which are called Oseledets subspaces and depend measurable on $x$ such that, for every $1 \leq i \leq l$,

- $\operatorname{dim}\left(E_{x}^{i, A}\right)$ is constant,

- $A(x) E_{x}^{i, A} \subseteq E_{f(x)}^{i, A}$ with equality when $\lambda_{i}>-\infty$

and

$$
\text { - } \lambda_{i}=\lim _{n \rightarrow+\infty} \frac{1}{n} \log \left\|A^{n}(x) v\right\| \text { for every non-zero } v \in E_{x}^{i, A} \text {. }
$$

This result extends a famous theorem due to Oseledets [Ose68] known as the multiplicative ergodic theorem which was originally stated in both, invertible (both $f$ and the matrices are assumed to be invertible) and non-invertible (neither $f$ nor the matrices are assumed to be invertible) settings (see also [Via14]). While in the invertible case the conclusion is similar to the conclusion above (except that all Lyapunov exponents are finite), in the non-invertible case, instead of a direct sum decomposition into invariant vector subspaces, one only get an invariant filtration (a sequence of nested subspaces) of $\mathbb{R}^{d}$.

Let us denote by

$$
\gamma_{1}(A, \mu) \geq \gamma_{2}(A, \mu) \geq \ldots \geq \gamma_{d}(A, \mu)
$$

the Lyapunov exponents of $(A, f)$ with respect to the measure $\mu$ counted with multiplicities. Given a periodic point $p$, we denote the Lyapunov exponents counted with multiplicities of $(A, f)$ at $p$ by $\left\{\gamma_{i}(A, p)\right\}_{i=1}^{d}$. When there is no risk of ambiguity, we suppress the index $A$ or even both $A$ and $\mu$ from the previous objects.

2.2. Angle between subspaces. The angle $\measuredangle(E, F)$ between two subspaces $E$ and $F$ of $\mathbb{R}^{d}$ is defined as follows: given $w \in \mathbb{R}^{d}$ we define

$$
\operatorname{dist}(w, E)=\inf _{v \in E}\|w-v\| \text {. }
$$


It is easy to see that $\operatorname{dist}(w, E)=\left\|w^{\perp}\right\|$ where $w^{\perp}=w-\operatorname{Proj}_{E} w$. More generally, we may consider the distance between $E$ and $F$ given by

$$
\operatorname{dist}(E, F)=\sup _{v \in E, w \in F}\left\{\operatorname{dist}\left(\frac{v}{\|v\|}, F\right), \operatorname{dist}\left(\frac{w}{\|w\|}, E\right)\right\} .
$$

Then, the angle between $E$ and $F$ is just $\measuredangle(E, F)=\sin ^{-1}(\operatorname{dist}(E, F))$.

2.3. Periodic approximation properties. We say that $(A, f, \mu)$ has the periodic approximation property for the Lyapunov exponents if there exists a sequence $\left(p_{k}\right)_{k \in \mathbb{N}}$ of periodic points satisfying

$$
\mu_{p_{k}}:=\frac{1}{n_{k}} \sum_{j=0}^{n_{k}-1} \delta_{f^{j}\left(p_{k}\right)} \underset{k \rightarrow \infty}{\stackrel{\text { weak }^{*}}{\longrightarrow}} \mu
$$

where $n_{k}$ is the $f$-period of $p_{k}$ and such that

$$
\gamma_{j}\left(A, p_{k}\right) \stackrel{k \rightarrow \infty}{\longrightarrow} \gamma_{j}(A, \mu)
$$

for every $j=1, \ldots, d$.

Similarly, $(A, f, \mu)$ is said to have the periodic approximation property for the Oseledets splitting if there exists a sequence $\left(p_{k}\right)_{k \in \mathbb{N}}$ of periodic points satisfying (3) and, moreover, for each $k \in \mathbb{N}$ there exists a set $\mathcal{G}_{k} \subset M$ with $\mu\left(\mathcal{G}_{k}\right)>1-\frac{1}{k}$ so that for every $x \in \mathcal{G}_{k}$ there exists $q \in \operatorname{orb}\left(p_{k}\right)$ satisfying

$$
\measuredangle\left(E_{x}^{j, A}, F_{q}^{j, A}\right)<\frac{1}{k}
$$

for every $j=1, \ldots, l$ where $F_{q}^{j, A}$ is the sum of Oseledets subspaces of $A$ at $q$ associated to consecutive Lyapunov exponents. More precisely, if $\gamma_{i_{1}}(A, \mu) \geq \cdots \geq$ $\gamma_{i_{t}}(A, \mu)$ are the Lyapunov exponents associated to $E_{x}^{j, A}$ then $F_{q}^{j, A}$ is the sum of the Oseledets subspaces associated to $\gamma_{i_{1}}\left(A, p_{k}\right) \geq \cdots \geq \gamma_{i_{t}}\left(A, p_{k}\right)$.

2.4. Main result. The main result of this note is the following one:

Theorem 2.1. Let $f: M \rightarrow M$ be a homeomorphism, $\mu$ an ergodic $f$-invariant probability measure and $A: M \rightarrow M(d, \mathbb{R})$ a continuous map. If the system $(A, f, \mu)$ has the periodic approximation property for the Lyapunov exponents then it also has the periodic approximation property for the Oseledets splitting.

In view of the previous result, it is natural then to ask whether the converse statement is also true. More precisely, if the system $(A, f, \mu)$ has the periodic approximation property for the Oseledets splitting then does it also have the periodic approximation property for the Lyapunov exponents? So far, we weren't able to prove neither to present a counter-example to it.

We say that $f$ satisfies the Anosov Closing property if there exist $C_{1}, \varepsilon_{0}, \theta>0$ such that if $z \in M$ satisfies $d\left(f^{n}(z), z\right)<\varepsilon_{0}$ then there exists a periodic point $p \in M$ such that $f^{n}(p)=p$ and

$$
d\left(f^{j}(z), f^{j}(p)\right) \leq C_{1} e^{-\theta \min \{j, n-j\}} d\left(f^{n}(z), z\right)
$$

for every $j=0,1, \ldots, n$. Examples of maps satisfying this property are shifts of finite type, basic pieces of Axiom A diffeomorphisms and more generally, hyperbolic homeomorphisms. See for instance, [KH95] p.269, Corollary 6.4.17. 
In what follows we are also going to assume that $A: M \rightarrow M(d, \mathbb{R})$ is an $\alpha$-Hölder continuous map. This means that there exists a constant $C_{2}>0$ such that

$$
\|A(x)-A(y)\| \leq C_{2} d(x, y)^{\alpha}
$$

for all $x, y \in M$ where $\|A\|$ denotes the operator norm of a matrix $A$, that is, $\|A\|=\sup \{\|A v\| /\|v\| ;\|v\| \neq 0\}$.

Corollary 2.2. Let $f: M \rightarrow M$ be a homeomorphism satisfying the Anosov Closing property, $\mu$ an ergodic $f$-invariant probability measure and $A: M \rightarrow M(d, \mathbb{R})$ an $\alpha$-Hölder continuous map. Then, $(A, f, \mu)$ has the periodic approximation property for the Lyapunov exponents and for the Oseledets splitting.

Proof. It follows from Theorem 2.1 of $[\mathrm{Bac}]$ and from its proof that $(A, f, \mu)$ has the periodic approximation property for the Lyapunov exponents. The result then follows applying Theorem 2.1.

As a simple consequence we have

Corollary 2.3. Let $(A, f, \mu)$ be as in the previous corollary. Then, for $\mu$-almost every $x \in M$ there exists a sequence of periodic points $\left(p_{k}\right)_{k \in \mathbb{N}}$ such that

$$
\measuredangle\left(E_{x}^{j, A}, F_{p_{k}}^{j, A}\right) \stackrel{k \rightarrow \infty}{\longrightarrow} 0
$$

for every $j=1, \ldots, l$. Moreover, the sequence $\left(p_{k}\right)_{k \in \mathbb{N}}$ may be taken so that

$$
\gamma_{j}\left(A, p_{k}\right) \stackrel{k \rightarrow \infty}{\longrightarrow} \gamma_{j}(A, \mu)
$$

for every $j=1, \ldots, d$ and

$$
\mu_{p_{k}}=\frac{1}{n_{k}} \sum_{j=0}^{n_{k}-1} \delta_{f^{j}\left(p_{k}\right)} \underset{k \rightarrow \infty}{\stackrel{w e a k^{*}}{\longrightarrow}} \mu
$$

where $n_{k}$ is the $f$-period of $p_{k}$.

\section{Preliminaries}

In this section we present some preliminary notions and results that are going to be used in the proof of our main theorem. We start by recalling the notion of semi-projective cocycle introduced in $[\mathrm{BP}]$.

3.1. Semi-projective cocycles. Let $\mathbb{P}^{d-1}$ denote the real $(d-1)$-dimensional projective space, that is, the space of all one-dimensional subspaces of $\mathbb{R}^{d}$. Given a continuous map $A: M \rightarrow M(d, \mathbb{R})$, we want to define an action on $\mathbb{P}^{d-1}$ which is, in some sense, induced by $A$. If $(x,[v]) \in M \times \mathbb{P}^{d-1}$ is such that $A(x) v \neq 0$ then we have a natural action induced by $A$ on $\mathbb{P}^{d-1}$ which is just given by $A(x)[v]=[A(x) v]$. The difficulty appears when $A(x) v=0$ for some $v \neq 0$. To bypass this issue, let us consider the closed set given by

$$
\operatorname{ker}(A)=\left\{(x,[v]) \in M \times \mathbb{P}^{d-1} ; A(x) v=0\right\} .
$$

If $\mu(\pi(\operatorname{ker}(A)))=0$ where $\pi: M \times \mathbb{P}^{d-1} \rightarrow M$ denotes the canonical projection on the first coordinate, then $A(x)$ is invertible for $\mu$-almost every $x \in M$ and hence it naturally induces a map on $\mathbb{P}^{d-1}$ which is defined $\mu$-almost everywhere and is all we need. Otherwise, if $\mu(\pi(\operatorname{ker}(A)))>0$ let us consider the set

$$
K(A)=\left\{(x,[v]) \in M \times \mathbb{P}^{d-1} ; A^{n}(x) v=0 \text { for some } n>0\right\} .
$$


Observe that $K(A) \cap\{x\} \times \mathbb{P}^{d-1} \subset\{x\} \times E_{x}^{l, A}$ for every regular point $x \in M$.

Since $\pi(K(A))$ is an $f$-invariant set and $\mu$ is ergodic it follows that $\mu(\pi(K(A)))=$ 1. Thus, we can define a mensurable section $\sigma: M \rightarrow \mathbb{P}^{d-1}$ such that $(x, \sigma(x)) \in$ $K(A)$. Moreover, we can do this in a way such that if $x \in \pi(\operatorname{ker}(A))$ then $(x, \sigma(x)) \in$ $\operatorname{ker}(A)$. Fix such a section. We now define the semi-projective cocycle associated to $A$ and $f$ as being the map $F_{A}: M \times \mathbb{P}^{d-1} \rightarrow M \times \mathbb{P}^{d-1}$ given by

$$
F_{A}(x,[v])=\left\{\begin{array}{l}
(f(x),[A(x) v]) \text { if } A(x) v \neq 0 \\
(f(x), \sigma(f(x)) \text { if } A(x) v=0 .
\end{array}\right.
$$

This is a measurable function which coincides with the usual projective cocycle outside $\operatorname{ker}(A)$. In particular, it is continuous outside $\operatorname{ker}(A)$. From now on, given a non-zero element $v \in \mathbb{R}^{d}$ we are going to use the same notation to denote its equivalence class in $\mathbb{P}^{d-1}$.

Given a measure $m$ on $M \times \mathbb{P}^{d-1}$, observe that if $m(\operatorname{ker}(A))=0$ then $F_{A *} m$ does not depend on the way the section $\sigma$ was chosen. Indeed, if $\psi: M \times \mathbb{P}^{d-1} \rightarrow \mathbb{R}$ is a mensurable function then

$$
\int_{M \times \mathbb{P}^{d-1}} \psi \circ F_{A} d m=\int_{M \times \mathbb{P}^{d-1} \backslash \operatorname{ker}(A)} \psi \circ F_{A} d m .
$$

In the sequel, we will be primarily interested in $F_{A}$-invariant measures on $M \times$ $\mathbb{P}^{d-1}$ projecting on $\mu$, that is, $\pi_{*} m=\mu$ and such that $m(\operatorname{ker}(A))=0$. We start by recalling a result from $[\mathrm{BP}]$ which says that if the cocycle $A$ has two different Lyapunov exponents then any such a measure may be written as a convex combination of measures concentrated on a suitable combination of the Oseledets subspaces. In order to state it, let us consider the Oseledets slow and fast subspaces of 'order i' associated to $A$ which are given, respectively, by

$$
E_{x}^{s_{i}, A}=E_{x}^{i+1, A} \oplus \cdots \oplus E_{x}^{l, A}
$$

and

$$
E_{x}^{u_{i}, A}=E_{x}^{1, A} \oplus \cdots \oplus E_{x}^{i, A}
$$

Proposition 3.1 (Propostion 3.1 of $[\mathrm{BP}]$ ). If $\gamma_{i}(A)>\gamma_{i+1}(A)$ then every $F_{A^{-}}$ invariant measure projecting on $\mu$ and such that $m(\operatorname{ker}(A))=0$ is of the form $m=a m^{u_{i}}+b m^{s_{i}}$ for some $a, b \in[0,1]$ such that $a+b=1$, where $m^{*}$ is an $F_{A^{-}}$ invariant measure projecting on $\mu$ such that its disintegration $\left\{m_{x}^{*}\right\}_{x \in M}$ with respect to $\mu$ satisfies $m_{x}^{*}\left(E_{x}^{*}\right)=1$ for $* \in\left\{s_{i}, u_{i}\right\}$.

3.2. The adjoint cocycle. Given $x \in M$, let $A^{*}(x):\left(\mathbb{R}^{d}\right)^{*} \rightarrow\left(\mathbb{R}^{d}\right)^{*}$ be the adjoint operator of $A\left(f^{-1}(x)\right)$ defined by

$$
\left(A^{*}(x) u\right) v=u\left(A\left(f^{-1}(x)\right) v\right) \text { for each } u \in\left(\mathbb{R}^{d}\right)^{*} \text { and } v \in \mathbb{R}^{d} .
$$

Fixing some inner product $\langle$,$\rangle on \mathbb{R}^{d}$ and identifying the dual space $\left(\mathbb{R}^{d}\right)^{*}$ with $\mathbb{R}^{d}$ we get the map $A^{*}: M \rightarrow M(d, \mathbb{R})$ and equation (5) becomes

$$
\left\langle A\left(f^{-1}(x)\right) u, v\right\rangle=\left\langle u, A^{*}(x) v\right\rangle \text { for every } u, v \in \mathbb{R}^{d} .
$$

The adjoint cocycle of $A$ is then defined as the cocycle generated by the map $A^{*}: M \rightarrow M(d, \mathbb{R})$ over $f^{-1}: M \rightarrow M$.

An useful remark is that the Lyapunov exponents counted with multiplicities of the adjoint cocycle are the same as those of the original cocycle. This follows from the fact that a matrix $B$ and its transpose $B^{T}$ have the same singular values combined with Kingman's sub-additive theorem. Moreover, Oseledets subspaces of 
the adjoint cocycle are strongly related with the ones of the original cocycle. More precisely, Lemma 3.1 of $[\mathrm{BP}]$ tells us that

$$
E_{x}^{s_{i}, A}=\left(E_{x}^{u_{i}, A^{*}}\right)^{\perp} \text { for every } i=1, \ldots, l
$$

where the right-hand side denotes the orthogonal complement of the space $E_{x}^{u_{i}}, A^{*}$.

3.3. Exterior powers and induced cocycles. For every $1 \leq j \leq d$ we denote by $\Lambda^{j}\left(\mathbb{R}^{d}\right)$ the $j$ th exterior power of $\mathbb{R}^{d}$ which is the space of alternate $j$-linear forms on the dual $\left(\mathbb{R}^{d}\right)^{*}$. If $\wedge$ denotes the exterior product of vectors of $\mathbb{R}^{d}$ then a basis for $\Lambda^{j}\left(\mathbb{R}^{d}\right)$ is given by $\left\{e_{i_{1}} \wedge \ldots \wedge e_{i_{j}} ; 1 \leq i_{1}<\ldots<i_{j} \leq l\right\}$ whenever $\left\{e_{i}\right\}_{i=1}^{d}$ is a basis for $\mathbb{R}^{d}$. We may also consider the exterior product $V \wedge W$ of subspaces $V$ and $W$ of $\mathbb{R}^{d}$. This is defined as the exterior product of the elements of any basis of $V$ with the elements of any basis of $W$. Any linear map $L \in \mathrm{M}(d, \mathbb{R})$ induces a linear map $\Lambda^{j} L: \Lambda^{j}\left(\mathbb{R}^{d}\right) \rightarrow \Lambda^{j}\left(\mathbb{R}^{d}\right)$ by

$$
\Lambda^{j} L(\omega): \phi_{1} \wedge \ldots \wedge \phi_{j} \rightarrow \phi_{1} \circ L \wedge \ldots \wedge \phi_{j} \circ L .
$$

Hence, a linear cocyle generated by $B: M \rightarrow M(d, \mathbb{R})$ over $f$ induces a linear cocycle over $f$ on the $j$ th exterior power which is generated by the map $x \rightarrow \Lambda^{j} B(x)$. Moreover, if $B$ satisfies the integrability condition so does $\Lambda^{j} B$ and its Lyapunov exponents are given by

$$
\left\{\gamma_{i_{1}}(B)+\ldots+\gamma_{i_{j}}(B) ; 1 \leq i_{1}<\ldots<i_{j} \leq l\right\} .
$$

Furthermore, Oseledets subspaces of $\Lambda^{j} B$ are strongly related with the ones of $B$. In particular, letting $x \in M$ be a regular point for $(B, f, \mu)$ and $d_{i}(B)=$ $\sum_{j=1}^{i} \operatorname{dim}\left(E_{x}^{j, A}\right)$ then for every $1 \leq i \leq l$ the Osleledets subspace of $\Lambda^{d_{i}(B)} B$ at the point $x \in M$ associated to $\gamma_{1}(B)+\gamma_{2}(B)+\ldots+\gamma_{d_{i}(B)}(B)$ is given by

$$
E_{x}^{1, B} \wedge \ldots \wedge E_{x}^{i, B} .
$$

This is all we are going to use about the Oseledets subspaces of induced cocycle.

Let $\operatorname{Grass}(j, d)$ denote Grassmannian manifold of $j$-dimensional subspaces of $\mathbb{R}^{d}$. The map $\psi: \operatorname{Grass}(j, d) \rightarrow \mathbb{P}\left(\Lambda^{j}\left(\mathbb{R}^{d}\right)\right)$ which assigns to each subspace $E \in$ $\operatorname{Grass}(j, d)$ the projective point $[v] \in \mathbb{P}\left(\Lambda^{j}\left(\mathbb{R}^{d}\right)\right)$, where $v=v_{1} \wedge \ldots \wedge v_{j}$ and $\left\{v_{1}, \ldots, v_{j}\right\}$ is any basis for $E$, is an embedding known as the Plücker embedding. Therefore, if $\rho(.,$.$) is a distance on \mathbb{P}\left(\Lambda^{j}\left(\mathbb{R}^{d}\right)\right)$ we may push it back to $\operatorname{Grass}(j, d)$ via $\psi$. More precisely, the map $\operatorname{dist}_{\Lambda^{j}\left(\mathbb{R}^{d}\right)}: \operatorname{Grass}(j, d) \times \operatorname{Grass}(j, d) \rightarrow \mathbb{R}$ given by

$$
\operatorname{dist}_{\Lambda^{j}\left(\mathbb{R}^{d}\right)}\left(E_{1}, E_{2}\right)=\rho\left(\psi\left(E_{1}\right), \psi\left(E_{2}\right)\right)
$$

is a distance on $\operatorname{Grass}(j, d)$ and moreover, if $\rho$ is a distance given by an inner product in the linear space $\Lambda^{j}\left(\mathbb{R}^{d}\right)$ then $\operatorname{dist}_{\Lambda^{j}\left(\mathbb{R}^{d}\right)}$ is equivalent to the distance defined in (2).

\section{Approximation of the fastest Oseledets subspace}

In this section we get the desired approximation property for $E_{x}^{1, A}$ whenever it has dimension one. We chose to present this case separately because its proof illustrates the main ideas used in the general case and, moreover, notations are simpler providing a cleaner exposition. 
Proposition 4.1. Assume $(A, f, \mu)$ has the periodic approximation property for the Lyapunov exponents and let $\left(p_{k}\right)_{k \in \mathbb{N}}$ be a sequence of periodic points satisfying (3) and (4). Assume also that $\operatorname{dim}\left(E_{x}^{1, A}\right)=1$. Then given $\varepsilon>0$ there exist an arbitrarily large $k \in \mathbb{N}$ and a set $\mathcal{G}^{1}:=\mathcal{G}_{\varepsilon}^{1} \subset M$ with $\mu\left(\mathcal{G}^{1}\right)>1-\varepsilon$ so that for every $x \in \mathcal{G}^{1}$ there exists $q \in \operatorname{orb}\left(p_{k}\right)$ satisfying

$$
\measuredangle\left(E_{x}^{1, A}, E_{q}^{1, A}\right)<\varepsilon .
$$

Proof. We start observing that as

$$
\gamma_{j}\left(A, p_{k}\right) \stackrel{k \rightarrow \infty}{\longrightarrow} \gamma_{j}(A, \mu)
$$

for every $j=1, \ldots, d$ and $\gamma_{1}(A, \mu)>\gamma_{2}(A, \mu)$ it follows that $\gamma_{1}\left(A, p_{k}\right)>\gamma_{2}\left(A, p_{k}\right)$ for every $k$ sufficiently large and thus $E_{p_{k}}^{1, A}$ is also one-dimensional. Let us assume without loss of generality that this is indeed the case for every $k \in \mathbb{N}$.

For each $k \in \mathbb{N}$, let us consider the measure

$$
m_{k}=\int_{M} \delta_{\left(x, E_{x}^{1, A}\right)} d \mu_{p_{k}}(x)
$$

and let $m$ be the measure given by

$$
m=\int_{M} \delta_{\left(x, E_{x}^{1, A}\right)} d \mu(x) .
$$

Observe that these are $F_{A}$-invariant measures on $M \times \mathbb{P}^{d-1}$ concentrated on $\left\{\left(x, E_{x}^{1, A}\right) ; x \in\right.$ $M\}$ and projecting to $\mu_{p_{k}}$ and $\mu$, respectively. Consequently, letting $\varphi_{A}: M \times$ $\mathbb{P}^{d-1} \rightarrow \mathbb{R}$ be the map given by

$$
\varphi_{A}(x, v)=\log \frac{\|A(x) v\|}{\|v\|},
$$

it follows easily from the definition and Birkhoff's ergodic theorem that

$$
\gamma_{1}\left(A, \mu_{p_{k}}\right)=\int_{M \times \mathbb{P}^{d-1}} \varphi_{A}(x, v) d m_{k}
$$

and

$$
\gamma_{1}(A, \mu)=\int_{M \times \mathbb{P}^{d-1}} \varphi_{A}(x, v) d m .
$$

We claim now that $m_{k}$ converges to $m$ in the weak* topology. Indeed, let $\left\{m_{k_{i}}\right\}_{i \in \mathbb{N}}$ be a convergent subsequence of $\left\{m_{k}\right\}_{k \in \mathbb{N}}$ and suppose it converges to $\tilde{m}$. Since $M \times \mathbb{P}^{d-1}$ is a compact space it suffices to prove that $\tilde{m}=m$. In order to do so, we need the following auxiliary result.

Lemma 4.2. The measure $\tilde{m}$ satisfies $\tilde{m}(\operatorname{ker}(A))=0$. Moreover, it is $F_{A}$-invariant.

Proof. Suppose by contradiction that $\tilde{m}(\operatorname{ker}(A))=2 c>0$. For each $\delta>0$ let us consider

$$
K_{\delta}=\left\{(x, v) \in M \times \mathbb{P}^{d-1} ;\left\|A(x) \frac{v}{\|v\|}\right\|<\delta\right\} .
$$

These are open sets such that $\operatorname{ker}(A)=\cap_{\delta>0} K_{\delta}$ and $\tilde{m}\left(K_{\delta}\right) \geq \tilde{m}(\operatorname{ker}(A))>c>0$.

Fix $b \in \mathbb{R}$ such that

$$
b<\gamma_{1}(A, \mu)-\sup _{x,\|v\|=1} \log \|A(x) v\|
$$

and let $\delta>0$ be such that $\log y<\frac{b}{c}$ for every $y<\delta$. Then, for every $i$ sufficiently large $m_{k_{i}}\left(K_{\delta}\right)>c>0$ and consequently 


$$
\begin{aligned}
\gamma_{1}\left(A, p_{k_{i}}\right) & =\int \varphi_{A} d m_{k_{i}}=\int_{K_{\delta}} \varphi_{A} d m_{k_{i}}+\int_{K_{\delta}^{c}} \varphi_{A} d m_{k_{i}} \\
& <b+\sup _{x,\|v\|=1} \log \|A(x) v\|
\end{aligned}
$$

contradicting the choice of $b$. Thus, $\tilde{m}(\operatorname{ker}(A))=0$ as we want.

To prove that $\tilde{m}$ is $F_{A}$-invariant one only has to show that, given a continuous map $\psi: M \times \mathbb{P}^{d-1} \rightarrow \mathbb{R}$,

$$
\lim _{i \rightarrow \infty} \int \psi \circ F_{A} d m_{k_{i}}=\int \psi \circ F_{A} d \tilde{m}
$$

Given $\varepsilon>0$ let $\delta>0$ be small enough so that $\tilde{m}\left(\overline{K_{\delta}}\right)<\frac{\varepsilon}{\|\psi\|}$. Let $\hat{\psi}: M \times \mathbb{P}^{d-1} \rightarrow$ $\mathbb{R}$ be a continuous function such that it coincides with $\psi \circ F_{A}$ outside $K_{\delta}$ and $\|\hat{\psi}\| \leq\|\psi\|$. Note that the existence of such a map is guaranteed by Tietze's extension theorem. Then,

$$
\begin{aligned}
\left|\int \psi \circ F_{A} d m_{k_{i}}-\int \psi \circ F_{A} d \tilde{m}\right| & \leq\left|\int_{K_{\delta}^{c}} \hat{\psi} d m_{k_{i}}-\int_{K_{\delta}^{c}} \hat{\psi} d \tilde{m}\right| \\
& +\int_{K_{\delta}}\left|\psi \circ F_{A}\right| d m_{k_{i}}+\int_{K_{\delta}}\left|\psi \circ F_{A}\right| d \tilde{m} \\
& <4 \varepsilon
\end{aligned}
$$

for every $i$ sufficiently large proving (11) and consequently the lemma.

Now, recalling that

$$
\gamma_{1}\left(A, p_{k_{i}}\right) \stackrel{i \rightarrow+\infty}{\longrightarrow} \gamma_{1}(A, \mu)
$$

and observing that

$$
\int_{M \times \mathbb{P}^{d-1}} \varphi_{A}(x, v) d m_{k_{i}} \stackrel{i \rightarrow+\infty}{\longrightarrow} \int_{M \times \mathbb{P}^{d-1}} \varphi_{A}(x, v) d \tilde{m}
$$

it follows from (9) that

$$
\gamma_{1}(A, \mu)=\int_{M \times \mathbb{P}^{d-1}} \varphi_{A}(x, v) d \tilde{m} .
$$

Thus, from Proposition 3.1 we get that $\tilde{m}=m$ as claimed. In fact, otherwise the referred proposition would give us that $\tilde{m}=a m^{1}+b m^{s}$ where $a, b \in(0,1)$ are such that $a+b=1$ and $m^{s}$ is an $F_{A}$-invariant measure concentrated on $\left\{\left(x, E_{x}^{2} \oplus \cdots \oplus\right.\right.$ $\left.\left.E_{x}^{l}\right) ; x \in M\right\}$. Therefore,

$$
\begin{aligned}
\gamma_{1}(A, \mu) & =\int_{M \times \mathbb{P}^{d-1}} \varphi_{A}(x, v) d \tilde{m} \\
& =a \int_{M \times \mathbb{P}^{d-1}} \varphi_{A}(x, v) d m^{1}+b \int_{M \times \mathbb{P}^{d-1}} \varphi_{A}(x, v) d m^{s} \\
& \leq a \gamma_{1}(A, \mu)+b \gamma_{2}(A, \mu)<\gamma_{1}(A, \mu) .
\end{aligned}
$$

Observe that as a consequence of this argument we also get that $m$ is the only $F_{A}$-invariant measure projecting on $\mu$ and satisfying (12). This is going to be used on Section 5 . 
Given $\varepsilon>0$, let $\mathcal{G}^{1} \subset \mathcal{R}^{\mu} \cap \operatorname{supp}(\mu)$ be a compact set satisfying $\mu\left(\mathcal{G}^{1}\right)>1-\varepsilon$ and such that

$$
x \rightarrow E_{x}^{1, A} \oplus \ldots \oplus E_{x}^{l, A}
$$

is continuous on $x \in \mathcal{G}^{1}$. Observe that the existence of such set is guaranteed by Lusin's theorem.

Fix $x \in \mathcal{G}^{1}$. Let $B\left(\left(x, E_{x}^{1, A}\right), \varepsilon\right)$ be the open $\varepsilon$-neighborhood of $\left(x, E_{x}^{1, A}\right)$ on $M \times \mathbb{P}^{d-1}$. Recall that we are considering $M \times \mathbb{P}^{d-1}$ endowed with the metric $\tilde{d}$ given by $\tilde{d}((y, v),(z, w))=d(y, z)+\measuredangle(v, w)$.

Thus, since $m\left(B\left(\left(x, E_{x}^{1, A}\right), \varepsilon\right)\right)>0$ (recall that $y \rightarrow E_{y}^{1, A}$ is continuous when restricted to an arbitrarily $\mu$-large set and $\mu$ gives positive measure to every open ball centered at $x)$ and $m_{k} \rightarrow m$ it follows that

$$
\liminf _{k \rightarrow \infty} m_{k}\left(B\left(\left(x, E_{x}^{1, A}\right), \varepsilon\right)\right) \geq m\left(B\left(\left(x, E_{x}^{1, A}\right), \varepsilon\right)\right)>0 .
$$

In particular, there exists $k_{0}(x) \in \mathbb{N}$ such that $m_{k}\left(B\left(\left(x, E_{x}^{1, A}\right), \varepsilon\right)\right)>0$ for every $k \geq k_{0}(x)$. Consequently, it follows from the definition of $m_{k}$ that for every $k \geq$ $k_{0}(x)$ there exists $j_{k} \in\left\{0,1, \ldots, n_{k}-1\right\}$ so that

$$
\measuredangle\left(E_{x}^{1, A}, E_{f^{j_{k}}\left(p_{k}\right)}^{1, A}\right)<\varepsilon .
$$

To conclude the proof it remains to observe that $k_{0}(x)$ may be taken independent of $x \in \mathcal{G}^{1}$. But this follows easily using that $\mathcal{G}^{1}$ is compact and the Oseledets splitting is continuous when restricted to it.

\section{Simultaneous approximations}

In this section we get the desired approximations for the Oseledets slow and fast subspaces. Recall the definitions of $E_{x}^{u_{j}, A}$ and $E_{x}^{s_{j}, A}$ given in Section 3.1.

Proposition 5.1. Assume $(A, f, \mu)$ has the periodic approximation property for the Lyapunov exponents and let $\left(p_{k}\right)_{k \in \mathbb{N}}$ be a sequence of periodic points satisfying (3) and (4). Then given $\varepsilon>0$ there exist an arbitrarily large $k \in \mathbb{N}$ and a set $\mathcal{G}^{s, u}:=\mathcal{G}_{\varepsilon}^{s, u} \subset M$ with $\mu\left(\mathcal{G}^{s, u}\right)>1-\varepsilon$ so that for every $x \in \mathcal{G}^{s, u}$ there exists $q \in \operatorname{orb}\left(p_{k}\right)$ satisfying

and

$$
\measuredangle\left(E_{x}^{u_{j}, A}, F_{q}^{u_{j}, A}\right)<\varepsilon
$$

$$
\measuredangle\left(E_{x}^{s_{j}, A}, F_{q}^{s_{j}, A}\right)<\varepsilon
$$

for every $j \in\{1, \ldots, l\}$.

Proof. We start observing that if $j=l$ then $E_{x}^{u_{l}, A}=\mathbb{R}^{d}=F_{q}^{u_{l}, A}$. So, we only have to prove the proposition for $j<l$.

For each $j=1, \ldots, l$ we set $d_{j}=\sum_{i=1}^{j} \operatorname{dim}\left(E_{x}^{i, A}\right)$ and similarly $d_{j}^{*}=\sum_{i=1}^{j} \operatorname{dim}\left(E_{x}^{i, A^{*}}\right)$. Let $\Lambda$ be the space

$$
\Lambda^{d_{1}}\left(\mathbb{R}^{d}\right) \times \Lambda^{d_{2}}\left(\mathbb{R}^{d}\right) \times \ldots \times \Lambda^{d_{l-1}}\left(\mathbb{R}^{d}\right) \times \Lambda^{d_{1}^{*}}\left(\mathbb{R}^{d}\right) \times \Lambda^{d_{2}^{*}}\left(\mathbb{R}^{d}\right) \times \ldots \times \Lambda^{d_{l-1}^{*}}\left(\mathbb{R}^{d}\right)
$$

and let $\mathbb{P}$ be equal to

$$
\mathbb{P}\left(\Lambda^{d_{1}}\left(\mathbb{R}^{d}\right)\right) \times \mathbb{P}\left(\Lambda^{d_{2}}\left(\mathbb{R}^{d}\right)\right) \times \ldots \times \mathbb{P}\left(\Lambda^{d_{l-1}}\left(\mathbb{R}^{d}\right)\right) \times \mathbb{P}\left(\Lambda^{d_{1}^{*}}\left(\mathbb{R}^{d}\right)\right) \times \mathbb{P}\left(\Lambda^{d_{2}^{*}}\left(\mathbb{R}^{d}\right)\right) \times \ldots \times \mathbb{P}\left(\Lambda^{d_{l-1}^{*}}\left(\mathbb{R}^{d}\right)\right) .
$$

The map $f_{\Lambda A}: M \times \Lambda \rightarrow M \times \Lambda$ which assigns to each point

$$
\left(x, v_{d_{1}}, \ldots, v_{d_{l-1}}, v_{d_{1}^{*}}, \ldots, v_{d_{l-1}^{*}}\right) \in M \times \Lambda
$$


the point

$\left(f(x), \Lambda^{d_{1}} A(x) v_{d_{1}}, \ldots, \Lambda^{d_{l-1}} A(x) v_{d_{l-1}}, \Lambda^{d_{1}^{*}} A^{*}(x) v_{d_{1}^{*}}, \ldots, \Lambda^{d_{l-1}^{*}} A^{*}(x) v_{d_{l-1}^{*}}\right) \in M \times \Lambda$ induces a semi-projective cocycle $F_{\Lambda A}: M \times \mathbb{P} \rightarrow M \times \mathbb{P}$ as described in Section 3.1 .

For each $k \in \mathbb{N}$, let us consider the measure

$$
m_{k}=\int_{M} \delta\left(x, E_{x}^{1, \Lambda^{d_{1}} A}, E_{x}^{1, \Lambda^{d_{2}} A}, \ldots, E_{x}^{1, \Lambda^{d_{l-1}} A}, E_{x}^{\left.1, \Lambda^{d_{1}^{*}} A^{*}, E_{x}^{1, \Lambda}{ }_{2}^{d_{2}^{*}} A^{*}, \ldots, E_{x}^{1, \Lambda}{ }^{d_{l-1}^{*} A^{*}}\right)} d \mu_{p_{k}}(x)\right.
$$

where $\mu_{p_{k}}$ is as in (3) and let $m$ be the measure given by

$$
m=\int_{M} \delta\left(x, E_{x}^{1, \Lambda^{d_{1}} A}, E_{x}^{1, \Lambda^{d_{2}} A}, \ldots, E_{x}^{1, \Lambda^{d_{l-1}} A}, E_{x}^{1, \Lambda_{1}^{*}} A^{*}, E_{x}^{\left.1, \Lambda^{d_{2}^{*}} A^{*}, \ldots, E_{x}^{1, \Lambda^{d_{l}^{*}-1} A^{*}}\right)} d \mu(x) .\right.
$$

Observe that from the choice of $d_{j}$ and $d_{j}^{*}$ and (7) and (8) these are well defined measures on $M \times \mathbb{P}$ and, moreover, they are $F_{\Lambda A}$-invariant measures concentrated on

$$
\left\{\left(x, E_{x}^{1, \Lambda^{d_{1}} A}, E_{x}^{1, \Lambda^{d_{2}} A}, \ldots, E_{x}^{1, \Lambda^{d_{l-1}} A}, E_{x}^{1, \Lambda^{d_{1}^{*}} A^{*}}, E_{x}^{1, \Lambda^{d_{2}^{*}} A^{*}}, \ldots, E_{x}^{1, \Lambda^{d_{l-1}^{*}} A^{*}}\right) ; x \in M\right\}
$$

and projecting to $\mu_{p_{k}}$ and $\mu$, respectively. Consequently, letting $\varphi_{\Lambda A}: M \times \mathbb{P} \rightarrow \mathbb{R}$ be the map given by

$$
\begin{aligned}
\varphi_{\Lambda A}\left(x, v_{d_{1}}, \ldots, v_{d_{l-1}}, v_{d_{1}^{*}}, \ldots, v_{d_{l-1}^{*}}\right) & =\log \frac{\left\|\Lambda^{d_{1}} A(x) v_{d_{1}}\right\|}{\left\|v_{d_{1}}\right\|}+\ldots+\log \frac{\| \Lambda^{d_{l-1} A(x) v_{d_{l-1}} \|}}{\left\|v_{d_{l-1}}\right\|} \\
& +\log \frac{\left\|\Lambda^{d_{1}^{*}} A^{*}(x) v_{d_{1}^{*}}\right\|}{\left\|v_{d_{1}^{*}}\right\|}+\ldots+\log \frac{\| \Lambda^{d_{l-1}^{*} A^{*}(x) v_{d_{l-1}^{*}} \|}}{\left\|v_{d_{l-1}^{*}}\right\|},
\end{aligned}
$$

it follows from the definition and Birkhoff's ergodic theorem that

$$
\int_{M \times \mathbb{P}} \varphi_{\Lambda A}\left(x, v_{d_{1}}, \ldots, v_{d_{l-1}}, v_{d_{1}^{*}}, \ldots, v_{d_{l-1}^{*}}\right) d m_{k}=2 \sum_{j=1}^{l-1} \gamma_{1}\left(\Lambda^{d_{j}} A, \mu_{p_{k}}\right)
$$

and

$$
\int_{M \times \mathbb{P}} \varphi_{\Lambda A}\left(x, v_{d_{1}}, \ldots, v_{d_{l-1}}, v_{d_{1}^{*}}, \ldots, v_{d_{l-1}^{*}}\right) d m=2 \sum_{j=1}^{l-1} \gamma_{1}\left(\Lambda^{d_{j}} A, \mu\right) .
$$

We now observe that $m_{k}$ converges to $m$ in the weak ${ }^{*}$ topology. Indeed, suppose $m_{k}$ converges to some measure $\tilde{m}$. Then, using the previous observations and proceeding as we did in the proof of Proposition 4.1 we get that $\tilde{m}$ is an $F_{\Lambda A^{-}}$ invariant measure on $M \times \mathbb{P}$ projecting to $\mu$ and satisfying

$$
\int_{M \times \mathbb{P}} \varphi_{\Lambda A}\left(x, v_{d_{1}}, \ldots, v_{d_{l-1}}, v_{d_{1}^{*}}, \ldots, v_{d_{l-1}^{*}}\right) d \tilde{m}=2 \sum_{j=1}^{l-1} \gamma_{1}\left(\Lambda^{d_{j}} A, \mu\right) .
$$

Now, the claim follows easily from our next result.

Lemma 5.2. If $\tilde{m}$ is an $F_{\Lambda A}$-invariant measure on $M \times \mathbb{P}$ projecting to $\mu$ such that

$$
\int_{M \times \mathbb{P}} \varphi_{\Lambda A}\left(x, v_{d_{1}}, \ldots, v_{d_{l-1}}, v_{d_{1}^{*}}, \ldots, v_{d_{l-1}^{*}}\right) d \tilde{m}=2 \sum_{j=1}^{l-1} \gamma_{1}\left(\Lambda^{d_{j}} A, \mu\right)
$$

then $\tilde{m}=m$.

In order to prove this lemma we are going to use the following simple fact 
Claim 5.3. Let $M_{1} \times M_{2}$ be a product space and for $j=1,2$ let $\pi_{j}: M_{1} \times M_{2} \rightarrow M_{j}$ be the canonical projection on $M_{j}$. If $\xi$ is a measure on $M_{1} \times M_{2}$ and $\left(\pi_{1}\right)_{*} \xi=\delta_{x_{1}}$ and $\left(\pi_{2}\right)_{*} \xi=\delta_{x_{2}}$ for some $x_{1} \in M_{1}$ and $x_{2} \in M_{2}$ then $\xi=\delta_{x_{1}} \times \delta_{x_{1}}$. Moreover, $a$ similar statement holds for measures on a product of any finite number of spaces.

Indeed, observing that

$$
1=\left(\pi_{1}\right)_{*} \xi\left(\left\{x_{1}\right\}\right)=\xi\left(\left\{x_{1}\right\} \times M_{2}\right)
$$

we get that supp $\xi \subset\left\{x_{1}\right\} \times M_{2}$. Similarly, we conclude that supp $\xi \subset M_{1} \times\left\{x_{2}\right\}$. Thus, supp $\xi \subset\left\{x_{1}\right\} \times\left\{x_{2}\right\}$ and $\xi=\delta_{x_{1}} \times \delta_{x_{2}}$.

Proof of Lemma 5.2. Let $\tilde{m}=\int_{M} \tilde{m}_{x} d \mu(x)$ be a disintegration of $\tilde{m}$ along $\{\{x\} \times$ $\mathbb{P}\}_{x \in M}$ and for each $j \in\{1, \ldots, l-1\}$ let $\pi_{j}: \mathbb{P} \rightarrow \mathbb{P}\left(\Lambda^{d_{j}}\left(\mathbb{R}^{d}\right)\right)$ and $\pi_{j}^{*}: \mathbb{P} \rightarrow$ $\mathbb{P}\left(\Lambda^{d_{j}^{*}}\left(\mathbb{R}^{d}\right)\right)$ be the canonical projections on $\mathbb{P}\left(\Lambda^{d_{j}}\left(\mathbb{R}^{d}\right)\right)$ and $\mathbb{P}\left(\Lambda^{d_{j}^{*}}\left(\mathbb{R}^{d}\right)\right)$, respectively, and $\nu_{x}^{j}=\left(\pi_{j}\right)_{*} \tilde{m}_{x}$ and $\nu_{x}^{j *}=\left(\pi_{j}^{*}\right)_{*} \tilde{m}_{x}$ be the projections of $\tilde{m}_{x}$ on $\mathbb{P}\left(\Lambda^{d_{j}}\left(\mathbb{R}^{d}\right)\right)$ and $\mathbb{P}\left(\Lambda^{d_{j}^{*}}\left(\mathbb{R}^{d}\right)\right)$, respectively. We claim now that, for each $j \in\{1, \ldots, l-1\}, \nu_{x}^{j}=$ $\delta_{E_{x}^{1, \Lambda^{d_{j}}}{ }^{\lambda_{A}}}$ and $\nu_{x}^{j *}=\delta_{E_{x}^{1, \Lambda^{d_{j}^{*}} A^{*}}}$ for $\mu$-almost every $x \in M$. Given $j \in\{1, \ldots, l-1\}$ let $F_{\Lambda^{d_{j} A}}$ denote the semi-projective cocycle induced by $\left(f, \Lambda^{d_{j}} A\right)$ on $M \times \mathbb{P}\left(\Lambda^{d_{j}}\left(\mathbb{R}^{d}\right)\right)$. Similarly we define $F_{\Lambda_{j}^{d_{j}^{*}} A^{*}}$.

Let us consider the measures

$$
\nu_{j}=\int_{M} \nu_{x}^{j} d \mu(x) \text { and } \nu_{j}^{*}=\int_{M} \nu_{x}^{j *} d \mu(x) .
$$

These are $F_{\Lambda^{d_{j}} A}$ and $F_{\Lambda_{j}^{d_{j}^{*}} A^{*}}$-invariant measures on $M \times \mathbb{P}\left(\Lambda^{d_{j}}\left(\mathbb{R}^{d}\right)\right)$ and $M \times$ $\mathbb{P}\left(\Lambda^{d_{j}^{*}}\left(\mathbb{R}^{d}\right)\right)$, respectively, projecting to $\mu$ and satisfying

$\gamma_{1}\left(\Lambda^{d_{j}} A, \mu\right)=\int \log \frac{\left\|\Lambda^{d_{j}} A(x) v_{d_{j}}\right\|}{\left\|v_{d_{j}}\right\|} d \nu^{j}$ and $\gamma_{1}\left(\Lambda^{d_{j}^{*}} A^{*}, \mu\right)=\int \log \frac{\left\|\Lambda^{d_{j}^{*}} A^{*}(x) v_{d_{j}^{*}}\right\|}{\left\|v_{d_{j}^{*}}\right\|} d \nu^{j *}$.

Indeed,

$$
\begin{aligned}
2 \sum_{j=1}^{l-1} \gamma_{1}\left(\Lambda^{d_{j}} A, \mu\right) & =\int_{M \times \mathbb{P}} \varphi_{\Lambda A}\left(x, v_{d_{1}}, \ldots, v_{d_{l-1}}, v_{d_{1}^{*}}, \ldots, v_{d_{l-1}^{*}}\right) d \tilde{m} \\
& =\int_{M} \int_{\mathbb{P}} \varphi_{\Lambda A}\left(x, v_{d_{1}}, \ldots, v_{d_{l-1}}, v_{d_{1}^{*}}, \ldots, v_{d_{l-1}^{*}}\right) d \tilde{m}_{x} d \mu(x) \\
& =\sum_{j=1}^{l-1} \int_{M} \int_{\mathbb{P}} \log \frac{\left\|\Lambda^{d_{j}} A(x) v_{d_{j}}\right\|}{\left\|v_{d_{j}}\right\|} d \tilde{m}_{x} d \mu(x) \\
& +\sum_{j=1}^{l-1} \int_{M} \int_{\mathbb{P}} \log \frac{\left\|\Lambda^{d_{j}^{*}} A^{*}(x) v_{d_{j}^{*}}\right\|}{\left\|v_{d_{j}^{*}}\right\|} d \tilde{m}_{x} d \mu(x) \\
& \leq \sum_{j=1}^{l-1} \int_{M} \int_{\mathbb{P}\left(\Lambda^{d_{j}}\left(\mathbb{R}^{d}\right)\right)} \log \frac{\left\|\Lambda^{d_{j}} A(x) v_{d_{j}}\right\|}{\left\|v_{d_{j}}\right\|} d \nu_{x}^{j} d \mu(x) \\
& +\sum_{j=1}^{l-1} \int_{M} \int_{\mathbb{P}\left(\Lambda^{d_{j}^{*}}\left(\mathbb{R}^{d}\right)\right)} \log \frac{\left\|\Lambda^{d_{j}^{*}} A^{*}(x) v_{d_{j}^{*}}\right\|}{\left\|v_{d_{j}^{*}}\right\|} d \nu_{x}^{j *} d \mu(x) .
\end{aligned}
$$


Thus, since

$$
\int_{M \times \mathbb{P}\left(\Lambda^{d_{j}}\left(\mathbb{R}^{d}\right)\right)} \log \frac{\left\|\Lambda^{d_{j}} A(x) v_{j}\right\|}{\left\|v_{j}\right\|} d \xi^{j} \leq \gamma_{1}\left(\Lambda^{d_{j}} A, \mu\right)
$$

for every $F_{\Lambda^{d_{j}} A}$-invariant measure $\xi^{j}$ projecting on $\mu$ and similarly for every $F_{\Lambda^{d_{j}^{*}} A^{*}}$ invariant measure projecting on $\mu$ our claim follows. Hence, from the uniqueness obtained in the proof of Proposition 4.1 we get that

$$
\nu_{j}=\int_{M} \delta_{\left(x, E_{x}^{1, \Lambda}{ }^{d_{j} A}\right)} d \mu(x) \text { and } \nu_{j}^{*}=\int_{M} \delta_{\left(x, E_{x}^{1, \Lambda}{ }^{d_{j}^{*}} A^{*}\right)} d \mu(x) .
$$

Consequently, since a disintegration is essentially unique we get that $\nu_{x}^{j}=\delta_{E_{x}^{1, \Lambda^{d_{j}} A}}$ and $\nu_{x}^{j *}=\delta_{E_{x}^{1, \Lambda}{ }_{j}^{d^{*}} A^{*}}$ for $\mu$-almost every $x \in M$ as claimed. Now, invoking Claim 5.3 we get that

$$
\begin{aligned}
& \tilde{m}_{x}=\delta_{E_{x}^{1, \Lambda} \Lambda_{1}^{d_{1}}} \times \delta_{E_{x}^{1, \Lambda} d_{2 A}} \times \ldots \times \delta_{E_{x}^{1, \Lambda}{ }^{d_{l-1}{ }^{j} A}} \times \delta_{E_{x}^{1, \Lambda} d_{1}^{*} A^{*}} \times \ldots \delta_{E_{x}^{1, \Lambda} d_{l-1}^{*} A^{*}} \\
& =\delta\left(x, E_{x}^{1, \Lambda^{d_{1}} A}, E_{x}^{1, \Lambda}{ }^{d_{2}} A, \ldots, E_{x}^{1, \Lambda^{d_{l}-1} A}, E_{x}^{1, \Lambda}{ }_{1}^{d_{1}^{*}} A^{*}, E_{x}^{1, \Lambda}{ }^{d_{2}^{*}} A^{*}, \ldots, E_{x}^{1, \Lambda}{ }_{l-1}^{d_{l}^{*}} A^{*}\right)
\end{aligned}
$$

for $\mu$-almost every $x \in M$ and thus $\tilde{m}=m$ as stated.

Now, using that $m_{k}$ converges to $m$ and proceeding as we did at the end of the proof of Proposition 4.1 we conclude that, given $\varepsilon^{\prime}>0$, there exist an arbitrarily large $k \in \mathbb{N}$ and a set $\mathcal{G}^{s, u}:=\mathcal{G}_{\varepsilon^{\prime}}^{s, u} \subset M$ with $\mu\left(\mathcal{G}^{s, u}\right)>1-\varepsilon^{\prime}$ so that for every $x \in \mathcal{G}^{s, u}$ there exists $q \in \operatorname{orb}\left(p_{k}\right)$ satisfying

$$
\measuredangle\left(E_{x}^{1, \Lambda^{d_{j}} A}, F_{q}^{1, \Lambda^{d_{j}} A}\right)<\varepsilon^{\prime}
$$

and

$$
\measuredangle\left(E_{x}^{1, \Lambda^{d_{j}^{*}} A_{*}}, F_{q}^{1, \Lambda^{d_{j}^{*}} A_{*}}\right)<\varepsilon^{\prime}
$$

for every $j \in\{1, \ldots, l-1\}$. Thus, recalling (8) we get that

$$
\measuredangle\left(E_{x}^{1, A} \wedge \ldots \wedge E_{x}^{j, A}, F_{q}^{1, A} \wedge \ldots \wedge F_{q}^{j, A}\right)<\varepsilon^{\prime}
$$

and

$$
\measuredangle\left(E_{x}^{1, A^{*}} \wedge \ldots \wedge E_{x}^{j, A^{*}}, F_{q}^{1, A^{*}} \wedge \ldots \wedge F_{q}^{j, A^{*}}\right)<\varepsilon^{\prime}
$$

for every $j \in\{1, \ldots, l-1\}$. Consequently, from the definition of $\operatorname{dist}_{\Lambda^{r}\left(\mathbb{R}^{d}\right)}$ it follows that

and

$$
\operatorname{dist}_{\Lambda^{r}\left(\mathbb{R}^{d}\right)}\left(E_{x}^{1, A} \oplus \ldots \oplus E_{x}^{j, A}, F_{q}^{1, A} \oplus \ldots \oplus F_{q}^{j, A}\right)<\varepsilon^{\prime}
$$

$$
\operatorname{dist}_{\Lambda^{r}\left(\mathbb{R}^{d}\right)}\left(E_{x}^{1, A^{*}} \oplus \ldots \oplus E_{x}^{j, A^{*}}, F_{q}^{1, A^{*}} \oplus \ldots \oplus F_{q}^{j, A^{*}}\right)<\varepsilon^{\prime}
$$

for every $j \in\{1, \ldots, l-1\}$. Now, using the fact that the distances $\operatorname{dist}_{\Lambda^{r}\left(\mathbb{R}^{d}\right)}$ and dist are equivalent and taking $\varepsilon^{\prime}>0$ smaller if necessary, it follows that

$$
\measuredangle\left(E_{x}^{1, A} \oplus \ldots \oplus E_{x}^{j, A}, F_{q}^{1, A} \oplus \ldots \oplus F_{q}^{j, A}\right)<\varepsilon
$$

and

$$
\measuredangle\left(E_{x}^{1, A^{*}} \oplus \ldots \oplus E_{x}^{j, A^{*}}, F_{q}^{1, A^{*}} \oplus \ldots \oplus F_{q}^{j, A^{*}}\right)<\varepsilon
$$

for every $j \in\{1, \ldots, l-1\}$. Summarizing, given $\varepsilon>0$ there exist an arbitrarily large $k \in \mathbb{N}$ and a set $\mathcal{G}^{s, u}:=\mathcal{G}_{\varepsilon}^{s, u} \subset M$ with $\mu\left(\mathcal{G}^{s, u}\right)>1-\varepsilon$ so that for every $x \in \mathcal{G}^{s, u}$ there exists $q \in \operatorname{orb}\left(p_{k}\right)$ satisfying

$$
\measuredangle\left(E_{x}^{u_{j}, A}, F_{q}^{u_{j}, A}\right)<\varepsilon
$$


and

$$
\measuredangle\left(E_{x}^{u_{j}, A^{*}}, F_{q}^{u_{j}, A^{*}}\right)<\varepsilon
$$

for every $j \in\{1, \ldots, l-1\}$. Thus, to conclude the proof of the proposition it remains to recall (6) which says that $E_{x}^{s_{j}, A}=\left(E^{u_{j}, A^{*}}\right)^{\perp}$.

\section{Conclusion of the proof}

Let $\left(p_{k}\right)_{k \in \mathbb{N}}$ be a sequence of periodic points satisfying (3) and (4). It is easy to see that in order to complete the proof of Theorem 2.1 it is enough to observe that given $\varepsilon>0$ there exist an arbitrarily large $k \in \mathbb{N}$ and a set $\mathcal{G}:=\mathcal{G}_{\varepsilon} \subset M$ with $\mu(\mathcal{G})>1-\varepsilon$ so that for every $x \in \mathcal{G}$ there exists $q \in \operatorname{orb}\left(p_{k}\right)$ satisfying

$$
\measuredangle\left(E_{x}^{j, A}, F_{q}^{j, A}\right)<\varepsilon
$$

for every $j=1, \ldots, l$. So, this is what we are going to do.

The cone of radius $\alpha>0$ around a subspace $V$ of $\mathbb{R}^{d}$ is defined as

$$
C_{\alpha}(V)=\left\{w_{1}+w_{2} \in V \oplus V^{\perp} ;\left\|w_{2}\right\|<\alpha\left\|w_{1}\right\|\right\} .
$$

Observe that this is equivalent to

$$
C_{\alpha}(V)=\left\{w \in \mathbb{R}^{d} ; \operatorname{dist}\left(\frac{w}{\|w\|}, V\right)<\alpha\right\}
$$

where dist is the distance defined in (2).

Lemma 6.1 (Lemma 4.2 of [BP]). Given $1 \leq j \leq l, \varepsilon^{\prime}>0$ and $\delta>0$ there exist a subset $K=K\left(\varepsilon^{\prime}\right) \subset M$ with $\mu(K)>1-\varepsilon^{\prime}$ and $\delta^{\prime}=\delta^{\prime}\left(\varepsilon^{\prime}, \delta\right) \in(0, \delta)$, such that for every $x \in K$,

$$
C_{\delta^{\prime}}\left(E_{x}^{u_{j}, A}\right) \cap C_{\delta^{\prime}}\left(E_{x}^{s_{j-1}, A}\right) \subset C_{\delta}\left(E_{x}^{j, A}\right) .
$$

Given $\varepsilon>0$, take $\varepsilon^{\prime}, \delta \in\left(0, \frac{\varepsilon}{10}\right)$ and let $K\left(\varepsilon^{\prime}\right) \subset M$ and $\delta^{\prime}>0$ be given by the previous lemma. Define $\mathcal{G}:=\mathcal{G}_{\delta^{\prime}}^{s, u} \cap K\left(\varepsilon^{\prime}\right)$ where $\mathcal{G}_{\delta^{\prime}}^{s, u}$ is the set associated to $\delta^{\prime}$ by Proposition 5.1. Then, $\mu(\mathcal{G})>1-\varepsilon$ and for every $x \in \mathcal{G}$ there exists $q \in \operatorname{orb}\left(p_{k}\right)$ satisfying

$$
\measuredangle\left(E_{x}^{u_{j}, A}, F_{q}^{u_{j}, A}\right)<\delta^{\prime}
$$

and

$$
\measuredangle\left(E_{x}^{s_{j}, A}, F_{q}^{s_{j}, A}\right)<\delta^{\prime}
$$

for every $j \in\{1, \ldots, l\}$ and moreover

$$
C_{\delta^{\prime}}\left(E_{x}^{u_{j}, A}\right) \cap C_{\delta^{\prime}}\left(E_{x}^{s_{j-1}, A}\right) \subset C_{\delta}\left(E_{x}^{j, A}\right) .
$$

Thus,

$$
F_{q}^{j, A} \subset F_{q}^{u_{j}, A} \cap F_{q}^{s_{j-1}, A} \subset C_{\delta^{\prime}}\left(E_{x}^{u_{j}, A}\right) \cap C_{\delta^{\prime}}\left(E_{x}^{s_{j-1}, A}\right) \subset C_{\delta}\left(E_{x}^{j, A}\right) .
$$

Consequently,

$$
\measuredangle\left(E_{x}^{j, A}, F_{q}^{j, A}\right)<\delta<\varepsilon
$$

for every $j \in\{1, \ldots, l\}$ as we wanted. The proof of Theorem 2.1 is now complete.

Acknowledgements. The author was partially supported by a CAPES-Brazil postdoctoral fellowship under Grant No. 88881.120218/2016-01 at the University of Chicago. 


\section{REFERENCES}

[Bac15] L. Backes, Rigidity of fiber bunched cocycles, Bulletin of the Brazilian Mathematical Society, 46 (2015), 163-179.

[Bac] L. Backes, On the periodic approximation of Lyapunov exponents for semi-invertible cocycles, Preprint https://arxiv.org/pdf/1612.04159.pdf

[BK16] L. Backes and A. Kocsard, Cohomology of dominated diffeomorphism-valued cocycles over hyperbolic systems, Ergodic Theory and Dynamical Systems, 36, (2016), 1703-1722.

[BP] L. Backes and M. Poletti. Continuity of Lyapunov exponents is equivalent to continuity of Oseledets subspaces. Stochastics and Dynamics, to appear.

[Bow75] R. Bowen, Equilibrium states and the ergodic theory of Anosov diffeomorphisms. Lecture Notes in Mathematics 470. Springer-Verlag, 1975.

[Dai10] X. Dai On the approximation of Lyapunov exponents and a question suggested by Anatole Katok, Nonlinearity, 23 (2010), 513-528.

[dLW10] R. de la Llave and A. Windsor, Livic theorems for non-commutative groups including diffeomorphism groups and results on the existence of conformal structur.es for Anosov systems, Ergodic Theory Dynam. Systems 30 (2010), 1055-1100

[DrF] D. Dragičević and G. Froyland, Hölder continuity of Oseledets splittings for semi-invertible operator cocycles, Ergod. Th. \& Dynam. Sys. To appear, doi: 10.1017/etds.2016.55

[FLQ10] G. Froyland, S. LLoyd, and A. Quas, Coherent structures and isolated spectrum for Perron-Frobenius cocycles, Ergod. Th. \& Dynam. Sys. 30 (2010), 729-756.

[Kal11] B. Kalinin, Livšic theorem for matrix cocycles. Annals of Mathematics, 173 (2011),10251042.

[KH95] A. Katok and B. Hasselblatt, Introduction to the modern theory of dynamical systems, Cambridge University Press, London-New York, 1995.

[KP16] A. Kocsard and R. Potrie, Livišic theorem for low-dimensional diffeomorphism cocycles, Commentarii Mathematici Helvetici, 91 (2016), 39-64.

[LLS14] C. Liang, G. Liao and W. Sun, A note on approximation properties of the Oseledets splitting, Proc. Amer. Math. Soc. 142 (2014), 3825-3838.

[LLS09] C. Liang, G. Liu and W. Sun, Approximation properties on invariant measure and Oseledec splitting in non-uniformly hyperbolic systems, Trans. Amer. Math. Soc. 361 (2009), 1543-1579.

[Liv71] A. Livšic. Homology properties of Y-systems, Math. Zametki 10 (1971), 758-763.

[Liv72] A. Livšic. Cohomology of dynamical systems, Math. USSR Izvestija 6 (1972), 1278-1301.

[Ose68] V. I. Oseledets, A multiplicative ergodic theorem: Lyapunov characteristic numbers for dynamical systems, Trans. Moscow Math. Soc. 19 (1968), 197-231.

[Sa15] V. Sadovskaya, Cohomology of fiber bunched cocycles over hyperbolic systems, Ergod. Th. \& Dynam. Sys. 35 (2015), 2669-2688.

[Sm67] S. Smale. Differentiable dynamical systems. Bull. Am. Math. Soc., 73:747-817, 1967.

[Via14] M. Viana, Lectures on Lyapunov Exponents, Cambridge University Press, 2014.

[WS10] Z. Wang and W. Sun, Lyapunov exponents of hyperbolic measures and hyperbolic periodic orbits, Trans. Amer. Math. Soc. 362 (2010), 4267-4282.

Departamento de Matemática, Universidade Federal do Rio Grande do Sul, Av. Bento Gonçalves 9500, CEP 91509-900, Porto Alegre, RS, Brazil.

E-MAIL: lhbackes@impa.br 